\title{
Práctica Académica para el Desarrollo Social en las Escuelas de Formación de la Policía Nacional de Colombia
}

\section{Academic Practice for Social Development in the Training Schools of the National Police of Colombia}

\author{
Jorman Estid García Sánchez \\ Universidad Católica de Colombia \\ Bogotá, Colombia \\ jorman.garcia@correo.policia.gov.co
}

Recibido: 27/08/2019 Aceptado: 26/11/2019

Resumen. En este artículo se presentan los fundamentos pedagógicos de una propuesta de ajuste al desarrollo de un línea de proyección social, prácticas académicas para el desarrollo social, dentro del Plan de Estudios del Técnico Profesional en Servicio de Policía, para el mejoramiento de la calidad del Modelo Nacional de Vigilancia Comunitaria por Cuadrantes (MNVCC) en la Policía Nacional de Colombia, como resultado de una investigación que propuso cambiar la forma de realizar la proyección social de los estudiantes que aspiran a ser nombrados policías, por lo cual el ajuste se propuso precisamente a la forma de realizar esta actividad en el proceso de formación policial, con fundamento en algunos aspectos pedagógicos encontrados dentro de la doctrina de la labor docente, establecida en la doctrina policial colombiana y en los resultados evidenciados en la investigación.

Es precisamente desde el Plan de Estudios del Programa Técnico Profesional en Servicio de Policía (TPSP) donde las actividades de proyección social se deben soportar y desarrollar, en temas de actualidad y acercamiento a la comunidad para el servicio policial. Por ende, se consideró que la asignatura propicia era la de Servicio de Policía, para que los estudiantes en su proceso de formación fortalezcan el acercamiento hacia la razón de ser de la Policía Nacional, que es nuestra ciudadanía colombiana y, como efecto en el campo laboral, se produzca un verdadero realce de la prevención y educación ciudadanas, aplicadas al Modelo Nacional de Vigilancia Comunitaria por Cuadrantes, que impactarían de una forma real desde las aulas al 
Revista Universidad en Diálogo • Vol. 10, N. ${ }^{\circ}$ 1, Enero-Junio, 2020 • 127-144

ISSN 2215-2849 • EISSN: 2215-4752

URL: http://www.revistas.una.ac.cr/index.php/dialogo/index CorReo ElECTRÓNICO: universidadendialogo@una.cr DOI: https://doi.org/10.15359/udre.10-1.8

servicio de la policía, fortaleciendo la habilidad para desarrollar su labor de manera segura y profesional.

El estudio se compone de tres aspectos importantes, en primer lugar, la relación entre las actividades de proyección social de la Dirección Nacional de Escuelas y las tareas de prevención y educación ciudadanas que se desarrollan en el marco del Modelo Nacional de Vigilancia Comunitaria por Cuadrantes; en segundo lugar, están las prácticas académicas para el desarrollo social dentro del plan curricular del Programa Técnico Profesional en Servicio de Policía; y como tercer aspecto se presenta la propuesta de fortalecimiento.

Palabras clave: fundamentos pedagógicos, prácticas académicas, proyección social, vigilancia comunitaria.

Abstract. This article describes the pedagogical foundations of a change proposal to develop a line of social projections, academic practices for social development, within the Curriculum of the Professional Technician in Police Service. This proposal aims to improve the quality of the National Model of Community Surveillance based on small geographical areas (MNVCC) the National Police of Colombia is implementing. The proposal resulted from a study that proposed changing how the social projection of students aspiring to be appointed as police officers is carried out. So, the object of the proposal is just to adjust how this activity is carried out in the police training process. The proposal is based on some pedagogical aspects found within the doctrine of teaching work, established in the Colombian police doctrine and the results evidenced in the research.

In fact, the activities of social projections must be supported and developed from the Curriculum of the Technical Professional Program in Police Service (TPSP), based on up-to-date information and community outreach for the police service. Therefore, the police service was considered as the appropriate subject for the students in their training process to strengthen their approach to the raison d'être of the National Police, which is our Colombian citizenship. Likewise, as an effect on the labor field, prevention, and citizen education, applied to the MNVCC, can be enhanced, and they can have a real impact, from the classrooms, on the police service, strengthening the ability to develop this service safely and professionally.

The study comprises three important aspects. The first one is the relationship between the social outreach activities of the National Directorate of Schools and the prevention and citizen education tasks carried out within the framework of the MNVCC. The second aspect is the academic practices for social development within the curricular plan of the Professional Technical Program in Police Service. The last one is the proposal for strengthening.

Keywords: pedagogical foundations, academic practices, social projection, community surveillance. 
URL: http://www.revistas.una.ac.cr/index.php/dialogo/index

CORREO ELECTRÓNICO: universidadendialogo@una.cr

DOI: https://doi.org/10.15359/udre.10-1.8

\section{Práctica Académica para el Desarrollo Social en las Escuelas de Formación de la Policía Nacional de Colombia}

Los policías íntegros y formados para la convivencia ciudadana son el resultado esperado de la formación académica de los ciudadanos seleccionados que aspiran a convertirse en profesionales de Policía, tema que la Policía Nacional de Colombia, a través de la Dirección Nacional de Escuelas, quiere tener como resultado de ese proceso profesional de capacitación que se imparte en las diferentes Escuelas de Policía, ubicadas en las diversas regiones del país, en las cuales se instruyen los patrulleros de la Policía Nacional, quienes obtienen el título de Técnico Profesional en Servicio de Policía (TPSP), programa académico único en Colombia, registrado en el Ministerio de Educación Nacional, que busca fortalecer con una formación integral a este personal, para afrontar las dinámicas sociales ante la exigente ciudadanía contemporánea, para la que los policías representan la visión de autoridad, de Estado y de ciudadanos ejemplares.

Para contribuir a ese propósito de la formación integral de los aspirantes a policías surgió una propuesta para esta clase de programa académico, en un momento en que la Dirección Nacional de Escuelas de la Policía Nacional (Dinae) está trabajando para ser reconocida como la primera universidad de policías en Colombia, haciendo parte de las pocas con que cuenta Latinoamérica, camino que debe darse con orientación a los tres pilares de la educación universitaria, como son la docencia, la investigación y la proyección social, tres pilares, de los cuales dos se observan adecuadamente soportados dentro de las dinámicas educativas de la Dinae, como son la docencia y la investigación, quedando la proyección social, que, a pesar de que está consagrada y en desarrollo a través de las escuelas de formación policial, se evidenció por medio de la investigación que existen debilidades en su práctica, a pesar de que la Policía Nacional tiene un gran escenario por su labor netamente social para desarrollarla mejor que cualquier universidad colombiana y que cuenta con un programa académico debidamente estructurado y certificado, pero donde no se encuentra plasmado cómo, con quién y bajo qué fundamento pedagógico los estudiantes y docentes deben realizar la proyección social.

\section{Actualidad de la proyección social en las Escuelas de Policía de Colombia}

La misión de la Dirección Nacional de Escuelas, según la Resolución No. 03856 del 07 de diciembre del 2009, "por la cual se define la estructura orgánica interna y se determinan las funciones de la Dirección Nacional de Escuelas", es direccionar la formación y capacitación integral del talento humano de la Policía Nacional, a través del Proyecto Educativo Institucional, en cumplimiento de las 
Revista Universidad en Diálogo • Vol. 10, N. ${ }^{\circ}$ 1, Enero-Junio, 2020 • 127-144

ISSN 2215-2849 • EISSN: 2215-4752

URL: http://www.revistas.una.ac.cr/index.php/dialogo/index CoRREO ELECTRÓNICO: universidadendialogo@una.cr DOI: https://doi.org/10.15359/udre.10-1.8

funciones de docencia, investigación y proyección social, con el fin de contribuir a la satisfacción de las necesidades de seguridad y convivencia ciudadana.

Las Escuelas de Policía tienen como misión desarrollar el Proyecto Educativo Institucional, a través de los procesos de formación, actualización y entrenamiento, así como capacitación e investigación, encargadas de operacionalizar los programas académicos dirigidos a la comunidad educativa, con el propósito de desarrollar competencias para el servicio de Policía (Policía Nacional de Colombia, 2009).

Ilustrado lo anterior, la investigación evidenció en primera instancia cómo se llevaba la proyección social en las Escuelas de Formación de la Policía Nacional de Colombia, en comparación con algunas universidades de profesiones liberales, en programas de Derecho, Administración de Empresas, Psicología, entre otros; a fin de aportar -luego de esa comparación y análisis de las dinámicas pedagógicas- una propuesta para mejorar esa función que se tiene planteada en la misión de la Dinae y para que la universidad de policía que se plantea ser en un futuro desarrolle una proyección social que realmente aporte a lo social y potencialice de manera significativa la formación de los hombres y las mujeres policías, impactando e incidiendo así en su forma de actuar y pensar frente a la comunidad y, por lo tanto, al servicio de policía que prestarán en el desarrollo de esta profesión.

\section{Líneas de proyección social en la Dirección Nacional de Escuelas}

En la investigación se exploró la función de la proyección social que plantea la Dirección Nacional de Escuelas de la Policía Nacional, la cual está fundamentada en la Resolución 000013 del 14 de enero de 2013, acto administrativo por medio del cual se expidieron y adoptaron los Lineamientos de Política en materia de proyección social en la Dirección Nacional de Escuelas de la Policía Nacional, definiendo esta como:

(...) una de las funciones esenciales de la Educación superior, que aporta al enriquecimiento de la labor docente e investigativa y dinamiza el conocimiento que el estudiantado construye en el espacio académico, tomando contacto con la realidad social a través de las prácticas sociales, encaminados a resolver determinados problemas de la comunidad y del entorno, logrando establecer estrechos vínculos con el sector externo, a través de la creación y ejecución de planes, programas y proyectos sociales que impactan la calidad de vida de la ciudadanía. 
URL: http://www.revistas.una.ac.cr/index.php/dialogo/index

CORREO ELECTRÓNICO: universidadendialogo@una.cr

DOI: https://doi.org/10.15359/udre.10-1.8

Se observó que existe desde el año 2009, mediante Resolución 03856 del mismo año 2009, la Vicerrectoría de Proyección Social, encargada de

Direccionar los programas que hagan partícipes de los beneficios de su actividad social, académica e investigativa; la promoción y desarrollo integral de un ser humano participativo en la construcción social, capaz de promover la calidad de vida de los ciudadanos a través de los programas de proyección social que propendan por la interacción con la comunidad; como también prestar apoyo y asesoría en los órdenes científicos tecnológicos, cultural y artístico y de seguridad ciudadana.

La cual cumple las siguientes funciones:

1. Propiciar y establecer procesos permanentes de interacción e integración con las comunidades nacionales e internacionales, en orden a promover su presencia en la vida social y cultural del país, a participar en el desarrollo de políticas nacionales y a contribuir a la comprensión y solución de los problemas más significativos.

2. Reconocer a los procesos de indagación y construcción de conocimiento en entornos sociales específicos; la contextualización e intercambio de experiencias y saberes; la formación y capacitación de la comunidad; y la socialización, difusión, promoción, circulación y comunicación del conocimiento científico, tecnológico, artístico y humanístico en la sociedad.

3. Construir procesos colectivos de desarrollo a partir de la interacción que la Dirección Nacional de Escuelas Policial tiene con los distintos actores y sectores de la sociedad.

4. Propender por el desarrollo del mantenimiento y mejora del sistema de gestión integral de la dependencia.

5. Las demás que le sean asignadas de acuerdo con la Ley y los reglamentos.

De allí que, para el cumplimiento de la misión de la Dinae, se establezca en la misma Resolución 03856 del año 2009, artículo 43, la existencia de unidades desconcentradas, encargadas de desarrollar los programas, bajo el direccionamiento de las vicerrectorías, facultades y demás dependencias encargadas de orientar la política educativa. Estas unidades son: 
1. Escuela de Cadetes de Policía "General Francisco de Paula Santander" (Ecsan)

2. Escuela dePostgrados de Policía “MiguelAntonio Lleras Pizarro"(Espol)

3. Escuela Nacional de Carabineros “Alfonso López Pumarejo"(Escar)

4. Centro de Instrucción Leticia (Cilet)

5. Escuela de Carabineros "Rafael Núñez" (Esran)

6. Escuela de Carabineros "Alejandro Gutiérrez" (Esagu)

7. Centro de Instrucción Pereira (Ciper)

8. Escuela de Carabineros "Eduardo Cuevas García” (Esecu)

9. Escuela de Carabineros Provincia de Vélez "Mayor General Manuel José López Gómez” (Esvel)

10.Escuela de Policía “Antonio Nariño" (Esana)

11.Escuela de Policía "Gabriel González" (Esgon)

12.Escuela de Policía "Rafael Reyes" (Esrey)

13.Escuela de Policía "Simón Bolívar" (Esbol)

14.Escuela de Policía Provincia de Sumapaz "Intendente Maritza Bonilla Ruiz" (Essum)

15.Escuela de Policía de Yuto "Miguel Antonio Caicedo Mena" (Esmac)

16.Escuela de Policía "Carlos Eugenio Restrepo" (Escer)

17.Escuela de Policía “Carlos Holguín Mallarino” (Escol)

18.Escuela de Aviación Policial (Esavi)

19.Escuela de Inteligencia y Contrainteligencia "Teniente Coronel Javier Antonio Uribe Uribe" (Escic)

20.Escuela de Investigación Criminal "Teniente Coronel Elkin Molina Aldana" (Esinc)

21.Escuela de Telemática y Electrónica "Teniente Coronel Jorge Luís Mauledoux Barón" (Estel) 
22.Escuela de Seguridad Vial "General Deogracias Fonseca Espinosa" (Esevi)

23.Escuela de Policía en Protección y Seguridad "Sargento Mayor Luís Alberto Torres Huertas" (Espro)

24.Escuela Internacional del Uso de la Fuerza Policial para la Paz (Cenop)

Cabe aclarar que dentro de las anteriores Escuelas de Formación Policial existen las Escuelas encargadas propiamente de la formación de patrulleros de la Policía Nacional, que hacen parte del nivel ejecutivo de esta Institución, quienes luego concursan para ingresar a ser mandos del mismo nivel ejecutivo en el grado de subintendente, intendente, intendente jefe, subcomisario y comisario, aquellos que de primera mano tienen el contacto con la comunidad en los cuadrantes del servicio de vigilancia en todo el territorio nacional, personal en algunos de esos grados mencionados, quienes aportaron en las encuestas a esta investigación. Las Escuelas de Formación son las siguientes:

1. Escuela Nacional de Carabineros "Alfonso López Pumarejo"(Escar)

2. Escuela de Carabineros "Rafael Núñez" (Esran)

3. Escuela de Carabineros "Alejandro Gutiérrez" (Esagu)

4. Escuela de Carabineros "Eduardo Cuevas García" (Esecu)

5. Escuela de Carabineros Provincia de Vélez "Mayor General Manuel José López Gómez" (Esvel)

6. Escuela de Policía "Antonio Nariño" (Esana)

7. Escuela de Policía "Carlos Eugenio Restrepo" (Escer)

8. Escuela de Policía "Gabriel González" (Esgon)

9. Escuela de Policía "Rafael Reyes" (Esrey)

10.Escuela de Policía “Simón Bolívar” (Esbol)

11.Escuela de Policía Provincia de Sumapaz "Intendente Maritza Bonilla Ruiz" (Essum)

12.Escuela de Policía de Yuto "Miguel Antonio Caicedo Mena"(Esmac) 
Revista Universidad en Diálogo • Vol. 10, N. ${ }^{\circ}$ 1, Enero-Junio, 2020 • 127-144

ISSN 2215-2849 • EISSN: 2215-4752

URL: http://www.revistas.una.ac.cr/index.php/dialogo/index CoRREO ELECTRÓNICO: universidadendialogo@una.cr

DOI: https://doi.org/10.15359/udre.10-1.8

De allí que en las Escuelas de Formación de la Dirección Nacional de Escuelas exista una dependencia encargada de realizar actividades de proyección social, liderada por la Vicerrectoría de Proyección Social, siguiendo lo dispuesto en la Resolución 000013 del 14 de enero de 2013, que plantea las siguientes líneas de proyección social, las cuales se ilustrarán a continuación:

Tabla 1

\begin{tabular}{|l|}
\hline \multicolumn{1}{|c|}{ Líneas de proyección social } \\
\hline Prácticas académicas para el desarrollo social: \\
Hace referencia al crecimiento y bienestar individual y/o colectivo que los \\
integrantes de las comunidades objeto pueden alcanzar a través de la intervención \\
social y educativa liderada por docentes y estudiantes policiales. \\
\hline Proyecto social para el emprendimiento: \\
Consiste en el apoyo, la orientación y la consultoría para que se direccionen \\
las propuestas comunitarias, encaminadas a la creación de microempresas, \\
cooperativas, empresas solidarias y demás iniciativas que satisfagan las \\
necesidades de la comunidad para el mejoramiento de su calidad de vida. \\
\hline Educación ciudadana: \\
A través de esta línea de acción se pretende contar con una población informada \\
para la vida personal, social y comunitaria, mediante la prevención y participación \\
consciente de sus derechos y obligaciones, solidaria y sensible con la sociedad. \\
\hline $\begin{array}{l}\text { Cultura, recreación y deportivo: } \\
\text { Es la línea que incluye las actividades de acercamiento con las comunidades } \\
\text { objeto, en donde se desarrollan programas y actividades culturales, artísticas, de } \\
\text { recreación y deporte. }\end{array}$ \\
\hline $\begin{array}{l}\text { Conservación y preservación del medio ambiente: } \\
\text { A través de las diferentes intervenciones sociales que realicen los estudiantes, se } \\
\text { propone educar y comprometer a la ciudadanía en la importancia de preservar y } \\
\text { conservar el medio ambiente, aportando al mejoramiento de la calidad de vida del } \\
\text { ecosistema. }\end{array}$ \\
\hline
\end{tabular}

Fuente: elaboración propia.

Una vez ubicados en el contexto que la Dirección Nacional de Escuelas (Dinae) tiene en cuanto a la proyección social en el programa que se desarrolla en las Escuelas de Formación Policial, como es el Técnico Profesional en Servicio de Policía, podemos adentrarnos en lo que propuso la investigación desarrollada en una de estas Escuelas de Policía, la Escuela de Policía Rafael 
URL: http://www.revistas.una.ac.cr/index.php/dialogo/index

CORREO ELECTRÓNICO: universidadendialogo@una.cr

DOI: https://doi.org/10.15359/udre.10-1.8

Reyes, ubicada en el municipio de Santa Rosa de Viterbo del departamento de Boyacá de la República de Colombia, tomando como punto de partida cómo se estaba desarrollando la proyección social y cómo desde una de las líneas establecidas por la Dirección Nacional de Escuelas para realizar esa proyección social, se puede proponer una nueva manera de desarrollar esta tarea misional de la educación, por parte de los estudiantes y docentes del (TPSP), buscando que se tenga clara una fundamentación pedagógica para ajustarla e incluirla dentro del plan de estudios de este programa técnico, para el mejoramiento de ciertas habilidades del futuro policía, que puedan ser aplicadas al salir graduados. Para ello se consideró pertinente que estos estudiantes se puedan enfrentar a escenarios reales controlados y en compañía de un docente, para así contribuir en impactar el modelo de seguridad ciudadana que hoy día tiene la Policía Nacional de Colombia, como es el Modelo Nacional de Vigilancia Comunitaria por cuadrantes (MNVCC).

Con los resultados de la investigación, se recuerda a las instituciones, y en particular a la Dinae, por su particular modelo de educación, la importancia que tiene la proyección social para la educación superior en el mundo y el impacto que dé una buena práctica de la misma, la cual influye en los estudiantes, los docentes y la comunidad, observándose que se presenta un aprendizaje en doble vía y significativo. Es por ello que surgió la pregunta: ¿En las Escuelas de la Policía Nacional de Colombia se estaba llevando de manera adecuada la proyección social?, y de no ser así, ¿cómo aportar para su mejora desde la academia, enfatizando en que las Escuelas de Formación Policial precisamente son instituciones universitarias con miras a convertirse en la primera universidad policial de Colombia?

Partiendo de una de las líneas de proyección social mencionada anteriormente, las prácticas académicas para el desarrollo social que deben ejercer los estudiantes en las Escuelas de Formación de la Dirección Nacional de Escuelas de la Policía Nacional, para la formación de patrulleros. Se realizó el análisis de los diferentes documentos sobre proyección social de la Dinae y de la Escuela de Policía Rafael Reyes, en cuanto a cómo se desarrolla esa línea de proyección social en la práctica, para luego compararla con el desarrollo de la proyección social de la Universidad Antonio Nariño del municipio de Duitama (Boyacá), institución que abrió las puertas a la investigación mediante la visita realizada por el investigador, mostrando la forma de hacer proyección social de los estudiantes de Derecho; igualmente se analizaron varios documentos de otras universidades encontrados en sus páginas oficiales, como fue la Universidad Abierta y a Distancia UNAD, con su programa de Psicología, la Universidad Externado de Colombia con su programa de Derecho, entre otras universidades colombianas, concluyendo lo siguiente: 
1. Se están realizando actividades en la Escuela de Formación Policial encaminadas a lo social, pero que no cuentan con la participación de todos los estudiantes del programa TPSP, en tanto en las universidades referenciadas sí se cuenta con tal participación.

2. Se evidenció que las actividades de proyección social de las Escuelas de Policía, si bien están soportadas en un acto administrativo, no están soportadas en el currículo propiamente dicho del programa, observándose actividades aisladas que muchas veces son desconocidas por los mismos estudiantes, docentes y, más aún, por los policías que laboran en los lugares donde se realizan estas actividades, ya en el campo profesional, situación que no sucede en las universidades de referencia, pues allí esa proyección social se da con una asignatura propiamente establecida, observando que se le da una importancia desde el currículo y los planes de trabajo, al igual que los estudiantes son conscientes de esa importancia, pues es una asignatura obligatoria.

3. Se observó también que estas actividades de proyección social les abren la puerta a los estudiantes a ese contacto directo con sus futuros clientes y los entornos sociales reales, beneficiándose la comunidad donde se desarrollan estas actividades de proyección social, todos los estudiantes de los programas o asignaturas y la universidad.

4. Se observa que la universidad compromete a la gran mayoría de docentes en la promoción de la proyección social y también se apoyan en ellos para la realización y supervisión de estas actividades, creándose una sinergia que le da importancia institucional al contacto directo con la comunidad y la práctica de lo aprendido por los estudiantes en un contexto real. Situación que en la Escuela de Formación Policial no está bien definida.

\section{Prácticas académicas para el desarrollo social}

Las prácticas académicas para el desarrollo social son la línea de proyección social que fue analizada y utilizada dentro de la investigación, puesto que su definición establece que "hace relación al crecimiento, y bienestar individual y/o colectivo que los integrantes de las comunidades objeto pueden alcanzar a través de la intervención social y educativa liderada por docentes y estudiantes policiales".

La línea de proyección social de prácticas académicas para el desarrollo social se observó entendida como el llevar a la praxis los conocimientos adquiridos en 
URL: http://www.revistas.una.ac.cr/index.php/dialogo/index

CORREO ELECTRÓNICO: universidadendialogo@una.cr

DOI: https://doi.org/10.15359/udre.10-1.8

las aulas, proyectarlos a una comunidad, en un terrero que asemeje la realidad laboral y que esa intervención social ocasione en alguna manera el crecimiento, el bienestar individual y/o colectivo de ese ciudadano o grupo de ciudadanos.

Al hablar de crecimiento y bienestar individual hacia la comunidad, se evidenció que la Policía Nacional de Colombia, desde mucho antes de que iniciara su idea de convertirse en universidad, en el desarrollo del servicio hacia la comunidad, tiene todo una oferta de servicios para propiciar ese crecimiento y bienestar comunitario, como los denominados Programas de Participación Ciudadana, entendidos en la práctica como aquellas herramientas de consolidación del Modelo Nacional de Vigilancia Comunitaria por Cuadrantes, ya que a través de un trabajo mancomunado entre autoridades y comunidad se proyecta posible el alcance seguridad y convivencia ciudadana en determinado sector; programas que deben ser desarrollados en la práctica por los integrantes de los cuadrantes de policía del Modelo Nacional de Vigilancia Comunitaria a lo largo y ancho del país.

Con una muestra representativa del personal que labora en el Modelo Nacional de Vigilancia Comunitaria por Cuadrantes, la investigación evidenció por medio de una encuesta, que existe una falta de acercamiento de los integrantes de la Policía a la comunidad, tendencia que también se observa en una encuesta realizada a nivel nacional por la empresa Datexco, donde la comunidad pide que la Policía Nacional de Colombia se acerque de nuevo.

La investigación fue desarrollada con una metodología de tipo mixto y los datos fueron recolectados y analizados a través de una estadística descriptiva, en tablas de contingencia de dos por dos, realizando un estudio de correlación, como lo describe Otero (2005),

En las tablas de contingencia, se exponen dos objetivos fundamentales, en primer lugar, organiza la información contenida en un experimento cuando ésta es de carácter bidimensional, es decir, cuando está referida a dos factores y con variables cualitativas, en segundo lugar a partir de la tabla de contingencia se puede además analizar si existe alguna relación de dependencia o independencia entre los niveles de las variables cualitativas, que precisamente son objeto de estudio para la investigación y en efecto dar respuesta a la pregunta que se trazó el autor.

En este caso, la pregunta que fue establecida en la investigación fue ¿cómo fortalecer la calidad del servicio de Policía mediante la estandarización de las tareas de proyección social, según el MNVCC, durante la formación de los TPSP, en búsqueda de la prevención y educación ciudadanas? 
Revista Universidad en Diálogo • Vol. 10, N. ${ }^{\circ}$ 1, Enero-Junio, 2020 • 127-144

ISSN 2215-2849 • EISSN: 2215-4752

URL: http://www.revistas.una.ac.cr/index.php/dialogo/index CoRREO ELECTRÓNICO: universidadendialogo@una.cr DOI: https://doi.org/10.15359/udre.10-1.8

Por consiguiente, se realizó una revisión teoría que contribuyó a orientar el camino y dar respuesta a este interrogante, principalmente en lo referente a los fundamentos pedagógicos, y al hablar de este, se recordó que es referirse a la necesidad de formar ciudadanos competentes (Area, 2012), situación que no es una búsqueda actual, sino de tiempos inmemoriales, donde surgieron preguntas como para qué se enseña, qué se debe enseñar y cómo se debe enseñar. De allí que la pedagogía, según Saviani (2013), debe ser llamada una superación de las teorías de la educación que incluyen teorías no críticas o pedagogía tradicional, nueva pedagogía y pedagogía tecnicista, por otra parte, las crítico-reproductivas o teoría de la escuela como violencia simbólica, teoría de la escuela dualista y la metodología de aprender haciendo (Ruiz, 2013).

Luego de realizar el anterior análisis de textos en materias de pedagogías, se observa que el desarrollo de la proyección social de las universidades referenciadas está fundamentado en la metodología de aprender haciendo. Según John Dewey (1859-1952), filósofo y educador norteamericano, correspondía a un programa de enseñanza práctico, ajustado a la experiencia de los estudiantes, implicando un hacer y una prueba (Ruiz, 2013); luego de instruido el estudiante en determinados conocimientos, estos deben ser llevados a la práctica en escenarios reales, en compañía de los docentes, pero donde son los mismos estudiantes quienes atienden a la comunidad y contribuyen a la solución de un problema, así se observa en la formación de los abogados en sus consultorios jurídicos y psicólogos en sus prácticas.

Es de resaltar que la formación por competencias constituye en la actualidad la base de la educación de la Policía Nacional, en donde los estudiantes desarrollan habilidades y destrezas que les faciliten desempeñarse exitosamente dentro del servicio policial; situación que hace completamente compatible que en la práctica de la proyección social se implemente un método claro de aprender haciendo, pero en los temas que serán objeto de desarrollo en la vida profesional y no en temas aislados y que los estudiantes no requerirán como tal en su ambiente práctico.

Hoy por hoy, la proyección social está concebida como un rol educativo, puesto que es idónea para retroalimentar, utilizando la praxis en este proceso de enseñanza-aprendizaje, promoviendo la incorporación en el currículo de las universidades de la región de la práctica de la pasantía universitaria, en ocasiones voluntaria, pero más de índole obligatoria (Fernández, 2013); por lo anterior, se considera que es propicio que el estudiante policía, quien desde su primer día de servicio en el ámbito profesional se enfrentará a la ciudadanía, experimente un proceso de enseñanza-aprendizaje-práctica, más aún cuando la Policía Nacional tiene escenarios en cada estación que pueden desarrollar perfectamente los temas de acercamiento comunitario que más se requieran. 
URL: http://www.revistas.una.ac.cr/index.php/dialogo/index

CORREO ELECTRÓNICO: universidadendialogo@una.cr

DOI: https://doi.org/10.15359/udre.10-1.8

Buscando esa relación entre los temas de proyección social que se dan en las Escuelas de Formación Policial y los Programas de Participación Ciudadana que impacten el servicio policial, se realizó un comparativo entre las líneas de proyección social dispuestas por la Dirección Nacional de Escuelas y los Programas de Participación Ciudadana que se desarrollan en el marco del MNVCC, así:

Tabla 2

\begin{tabular}{|c|c|}
\hline $\begin{array}{l}\text { Líneas de proyección social } \\
\text { Dinae (Dirección Nacional de Escuelas }\end{array}$ & $\begin{array}{l}\text { Programas de Participación Ciudadana } \\
\text { Disec (Dirección de Seguridad Ciudadana) }\end{array}$ \\
\hline $\begin{array}{l}\text { Prácticas académicas para el desarrollo social: } \\
\text { Tiene relación con el crecimiento y el bienestar individual } \\
\text { y/o colectivo que los integrantes de las comunidades } \\
\text { objeto pueden alcanzar a través de la intervención social y } \\
\text { educativa liderada por docentes y estudiantes policiales. }\end{array}$ & $\begin{array}{l}\text { Realizar escuelas de convivencia y } \\
\text { seguridad ciudadana } \\
\text {. Crear y fortalecer frentes de seguridad }\end{array}$ \\
\hline $\begin{array}{l}\text { Proyecto social para el emprendimiento: } \\
\text { Consiste en el apoyo, orientación y consultoría para } \\
\text { que se direccionen las propuestas comunitarias, } \\
\text { encaminadas a la creación de microempresas, } \\
\text { cooperativas, empresas solidarias y demás iniciativas } \\
\text { que satisfagan las necesidades de la comunidad para el } \\
\text { mejoramiento de su calidad de vida. }\end{array}$ & . Asesorar proyectos productivos en el área rural \\
\hline $\begin{array}{l}\text { Educación ciudadana: } \\
\text { A través de esta línea de acción se pretende contar con } \\
\text { una población informada para la vida personal, social } \\
\text { y comunitaria, mediante la prevención y participación, } \\
\text { consciente de sus derechos y obligaciones, solidaria y } \\
\text { sensible con la sociedad. }\end{array}$ & $\begin{array}{l}\text { Los frentes de seguridad ciudadana } \\
\text {. Realizar encuentros comunitarios } \\
\text {. Programa Policía Cívica de Mayores }\end{array}$ \\
\hline $\begin{array}{l}\text { Cultura, recreación y deporte: } \\
\text { Es la línea que incluye las actividades de acercamiento } \\
\text { con las comunidades objeto, en donde se desarrollan } \\
\text { programas y actividades culturales, artísticas, de } \\
\text { recreación y deporte. }\end{array}$ & $\begin{array}{l}\text { Programas Jóvenes a lo Bien } \\
\text {. Programa Cívica Infantil y Juvenil }\end{array}$ \\
\hline $\begin{array}{l}\text { Conservación y preservación del medio ambiente: } \\
\text { A través de las diferentes intervenciones sociales } \\
\text { que realicen los estudiantes, se propone educar y } \\
\text { comprometer a la ciudadanía en la importancia de } \\
\text { preservar y conservar el medio ambiente, aportando al } \\
\text { mejoramiento de la calidad de vida del ecosistema. }\end{array}$ & $\begin{array}{l}\text { Programa Cívica Infantil y Juvenil } \\
\text { Programas Jóvenes a lo Bien }\end{array}$ \\
\hline
\end{tabular}

Fuente: elaboración propia. 
Este cuadro comparativo mostró la relación entre las líneas de proyección social con las cuales se está desarrollando la proyección social en las Escuelas Policiales y la relación con los Programas de Participación que se desarrollan en el campo profesional del servicio de Policía, por lo cual se propuso en la línea de proyección social de prácticas académicas (el método aprender haciendo) que se realice el ajuste pertinente para que los Programas de Participación Ciudadana sean expuestos con importancia en la asignatura de Servicio de Policía y se programen con todos los estudiantes salidas para que estos temas se realicen en la práctica, en cualquier Estación de Policía que quiera afianzar el acercamiento a la comunidad, con la compañía de los docentes policiales y los mismos integrantes de los cuadrantes; estas mismas actividades se podrían dar en cualquier institución universitaria que quiera que sus estudiantes practiquen lo que les enseñan, para que realmente se realice esa proyección hacia la sociedad.

\section{Respecto a la propuesta de ajuste para hacer proyección social}

Luego de realizar una encuesta al personal de una Estación de Policía, se pudo evidenciar los siguientes resultados ( 1 es completamente de acuerdo, 5 es completamente en desacuerdo).

Las pedagogía recibida en la Escuela de formación para acercarme a la comunidad fue la adecuada
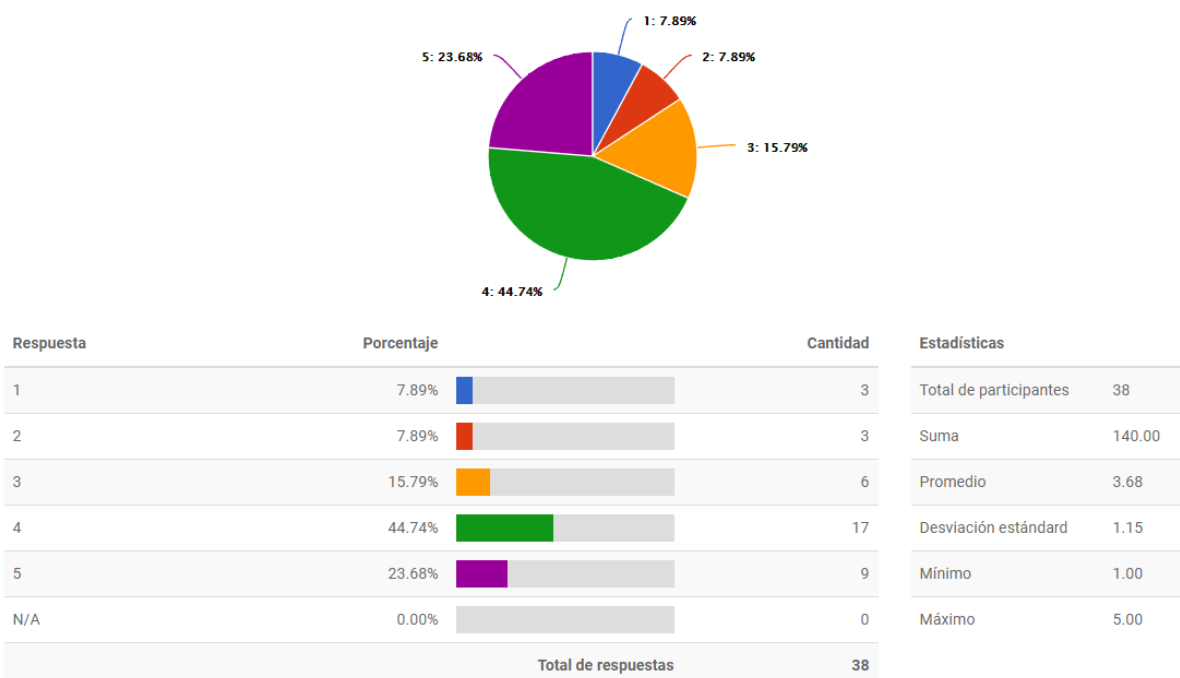

Figura 1. La pedagogía recibida en la Escuela de Formación para acercarme a la comunidad fue la adecuada. Fuente: elaboración propia 
URL: http://www.revistas.una.ac.cr/index.php/dialogo/index

CORREO ELECTRÓNICO: universidadendialogo@una.cr

DOI: https://doi.org/10.15359/udre.10-1.8

Mis superiores hablan y conocen los programas de participación ciudadana que oferta la Policía Nacional

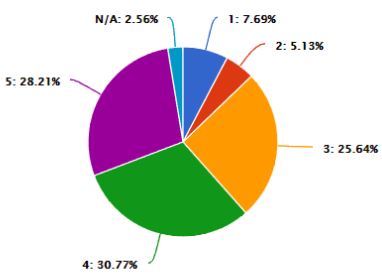

\begin{tabular}{lrrr} 
Respuesta & Porcentaje & Cantidad \\
\hline 1 & $7.69 \%$ & 3 \\
\hline 2 & $5.13 \%$ & 2 \\
\hline 3 & $25.64 \%$ & 10 \\
\hline 4 & $30.77 \%$ & & 12 \\
\hline 5 & $28.21 \%$ & & 11 \\
N/A & $2.56 \%$ & & 39
\end{tabular}

\begin{tabular}{ll} 
Estadisticas \\
\hline Total de participantes & 39 \\
\hline Suma & 140.00 \\
\hline Promedio & 3.68 \\
\hline Desviación estándard & 1.17 \\
\hline Mínimo & 1.00 \\
\hline Máximo & 5.00 \\
\hline
\end{tabular}

Figura 2. Mis superiores hablan y conocen los Programas de Participación Ciudadana que oferta la Policía Nacional. Fuente: elaboración propia.

Aporto a los funcionarios de PRECI (Prevención Ciudadana) de mi Estación y desarrollo algún programa de participación ciudadana

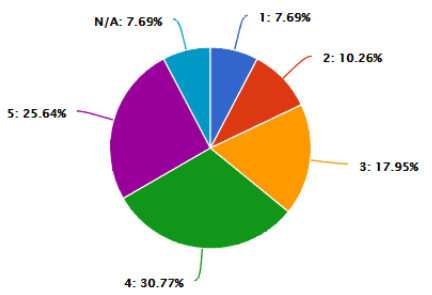

\begin{tabular}{|c|c|c|c|c|}
\hline Respuesta & Porcentaje & Cantidad & Estadísticas & \\
\hline 1 & $7.69 \%$ & 3 & Total de participantes & 39 \\
\hline 2 & $10.26 \%$ & 4 & Suma & 130.0 \\
\hline 3 & $17.95 \%$ & 7 & Promedio & 3.61 \\
\hline 4 & $30.77 \%$ & 12 & Desviación estándard & 1.23 \\
\hline 5 & $25.64 \%$ & 10 & Mínimo & 1.00 \\
\hline$N / A$ & $7.69 \%$ & 3 & Máximo & 5.00 \\
\hline & & 39 & & \\
\hline
\end{tabular}

Figura 3. Aporto a los funcionarios de Preci (Prevención Ciudadana) de mi Estación y desarrollo algún Programa de Participación Ciudadana. Fuente: elaboración propia. 
Revista Universidad en Diálogo • Vol. 10, N. ${ }^{\circ}$ 1, Enero-Junio, 2020 • 127-144

ISSN 2215-2849 • EISSN: 2215-4752

URL: http://www.revistas.una.ac.cr/index.php/dialogo/index

CORREO ELECTRÓNICO: universidadendialogo@una.cr

DOI: https://doi.org/10.15359/udre.10-1.8

Egresé de la Escuela de formación con unas buenas herramientas en materia de prevención para cumplir el servicio en el MNVCC

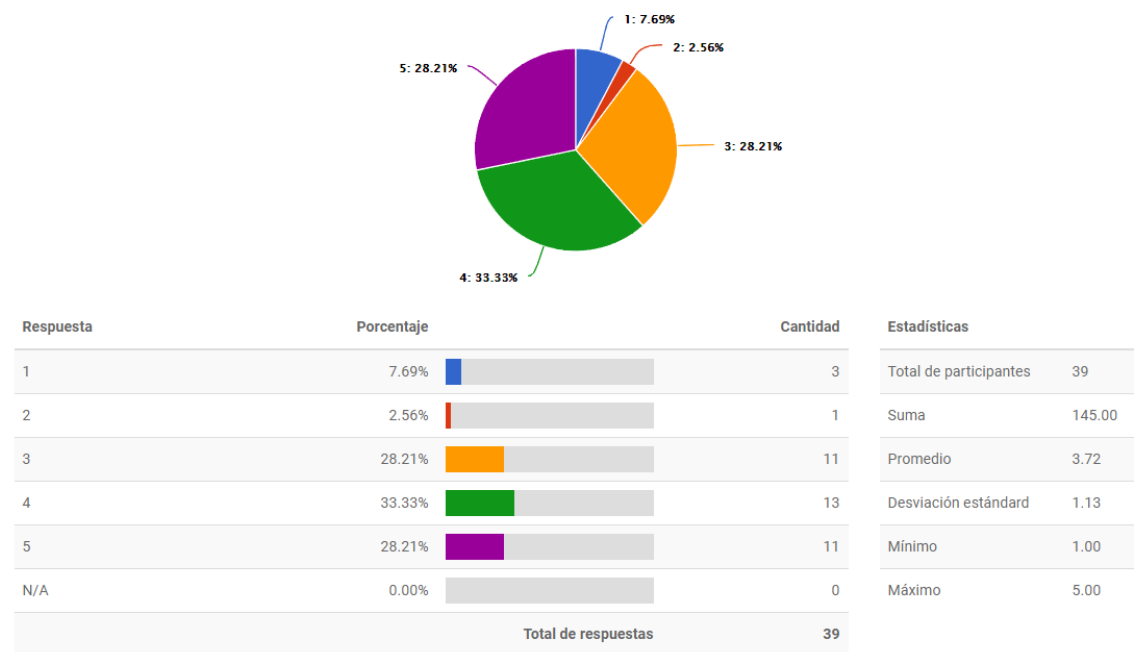

Figura 4. Egresé de la Escuela de Formación con unas buenas herramientas en materia de prevención para cumplir el servicio en el MNVCC. Fuente: elaboración propia

Tengo seguridad al momento de proponer que programa de participación ciudadana voy a utilizar en determinado problema en mi cuadrante

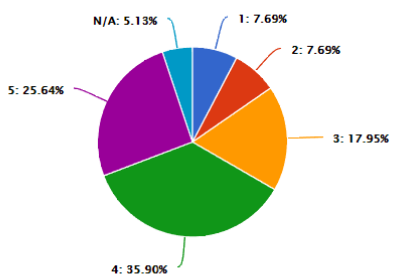

\begin{tabular}{|c|c|c|c|c|}
\hline Respuesta & Porcentaje & Cantidad & Estadisticas & \\
\hline 1 & $7.69 \%$ & 3 & Total de participantes & 39 \\
\hline 2 & $7.69 \%$ & 3 & Suma & 136.00 \\
\hline 3 & $17.95 \%$ & 7 & Promedio & 3.68 \\
\hline 4 & $35.90 \%$ & 14 & Desviación estándard & 1.19 \\
\hline 5 & $25.64 \%$ & 10 & Minimo & 1.00 \\
\hline $\mathrm{N} / \mathrm{A}$ & $5.13 \%$ & 2 & Máximo & 5.00 \\
\hline & & 39 & & \\
\hline
\end{tabular}

Figura 5. Tengo seguridad al momento de proponer cuál Programa de Participación Ciudadana voy a utilizar en determinado problema en mi cuadrante. Fuente: elaboración propia 
URL: http://www.revistas.una.ac.cr/index.php/dialogo/index

CORREO ELECTRÓNICO: universidadendialogo@una.cr

DOI: https://doi.org/10.15359/udre.10-1.8

$\mathrm{Al}$ realizar un análisis de las encuestas en su conjunto, las cuales fueron desarrolladas para medir si el personal de egresados del programa Técnico Profesional de la Escuela de Formación está familiarizado con los Programas de Participación Ciudadana en su contexto laboral y en su época de estudiantes, pudiendo observar que el resultado debería estar inclinado al 1 o 2 , significando estar de acuerdo, pero se observa que las respuestas se inclinan de 3 a 5 , mostrando una debilidad en este aspecto que refleja directamente qué tan sintonizado está el servidor policial para interactuar con la ciudadanía, resultados validados por la misma encuesta a nivel nacional realizada por la empresa Datexco, en la cual la ciudadanía pide esa cercanía del policía con la comunidad.

Es por lo anterior que la investigación encontró una relación directa entre la falta de práctica en el entorno de los estudiantes de los conocimientos dados, el no desarrollo adecuado de la proyección social y el no acercamiento del policía a la comunidad luego de egresado de la Escuela de Formación, puesto que no se le da esa importancia curricular que deberían tener los Programas de Participación Ciudadana y esa oportunidad de convertirlos en herramientas de proyección social, más aún cuando el servicio de policía, en su forma esencial, se presta en las calles en un ámbito de interacción preventiva y educativa.

\section{Conclusiones}

Este artículo evidencia primeramente a nivel general que el proceso de educación es un proceso en constante cambio y se debe adaptar a las exigencias de la razón de ser de cada programa que brinden las instituciones educativas y más aquellas universitarias, buscando siempre la mejor forma de que los estudiantes realmente aprendan y sean competentes para lo que desarrollarán y se emplearán luego de su egreso de las mismas; quiere decir que lo dado en las aulas serán herramientas apropiadas para su práctica profesional y que vayan directamente relacionadas al objeto de la empresa o institución en su campo determinado.

En la investigación se pudo observar la importancia que la proyección social tiene en las instituciones universitarias, siendo una responsabilidad social iluminar a la comunidad y una oportunidad para que los estudiantes se enfrenten desde su aprendizaje con sus futuros clientes, para que de esta forma desarrollen el conocimiento adquirido, con la metodología aprender haciendo, puesto que es una metodología educativa que ha sido desarrollada por varias instituciones y trae muy buenos resultados, dotando de seguridad y herramientas reales a los estudiantes.

Las Escuelas de Formación Policial tienen la oportunidad de realizar una proyección social en escenarios reales, con programas que se están aplicando por 
los hombres y las mujeres policías en su servicio habitual, puesto que se evidenciaron debilidades en las Escuelas en cuanto a las herramientas y la práctica del acercamiento o interacción comunitaria que están afectando la percepción de la comunidad para con la Institución, situaciones que llevan consigo el ajuste del currículo y demás documentos para contrarrestar estas falencias identificadas.

En las instituciones de educación superior es necesario mirar a sus egresados ya en el terrero, preguntar por aquellos aspectos relevantes o por las herramientas que fueron dadas en las clases o aquellas que se ausentaron, para así realizar los ajustes necesarios para que se pueda dar el mejoramiento continuo y que las instituciones entreguen a la sociedad estos profesionales competentes que requieren las comunidades.

\section{Referencias bibliográficas}

Fernández, C. M. (2013). ¿Extensión universitaria, proyección social o tercera misión? Revista Congreso Universidad, 2(2), 1-11.

García, J. E. (2018). Fundamentos pedagógicos de una propuesta de ajuste a la línea de proyección social: "prácticas académicas para el desarrollo social", dentro del plan de estudios del TPSP, para el mejoramiento de la calidad del Modelo Nacional de Vigilancia Comunitaria. Escuela de Policía Nacional, Boyacá, Santa Rosa de Viterbo.

Manuel Area, A. G. (2012). La alfabetización informacional y digital: fundamentos pedagógicos para la enseñanza. Revista Española de Documentación Científica, 46-74

Otero, J. V. (2005). Las tablas de contingencia. https://www.myheritage.es/ search-records?action $=$ person\&siteId $=68915281 \&$ indId $=1000004 \&$ or igin=profile: $\mathrm{https}: / / \mathrm{www}$.myheritage.es/search-records?action=person \&siteId=68915281\&indId=1000004\&origin=profile

Policía Nacional de Colombia (2009). Doctrina educativa para el docente policial. Bogotá: Imprenta Nacional de Colombia.

Ruiz, G. (2013). La teoría de la experiencia de John Dewey: significación histórica y vigencia en el debate histórico contemporáneo. Foro de Educación, 11(15),103-124. http://dx.doi.org/10.14516/fde.2013.011.015.005

Saviani, D. (2013). La pedagogía histórica crítica. Barão Geraldo: Autores Asociados limitada. 